\title{
Importance of Surgical Delay on Mortality in Patients with Hip Fracture: Mini-Review
}

\author{
Tarazona Santabalbina $F J^{* 1,2}$, Cuesta Peredó $D^{2,3}$, Belenguer Varea $A^{1,2}$, De la Camara JM${ }^{4}$, Sivera $S^{5}$ and \\ Avellana Zaragoza JA $\mathbf{A}^{1,2}$ \\ ${ }^{1}$ Department of Geriatric Medicine, Hospital Universitario de la Ribera, Spain
}

${ }^{2}$ Medical School, Universidad Católica de Valencia San vicente Mártir, Spain

${ }^{3}$ Department of Heath Care Quality, Hospital Universitario de la Ribera, Spain

${ }^{4}$ Department for Bibliometrics and Publication, Hospital Universitario de la Ribera, Spain.

${ }^{5}$ Department of Anesthesia, Hospital Universitario de la Ribera, Spain.

Received: March 28, 2018; Published: April 11, 2018

*Corresponding author: Francisco José Tarazona-Santabalbina, Hospital Universitario de la Ribera, Carretera de Corbera, E46600 Alzira, Valencia, Spain, Tel: 34962458 387; Fax: +34 962458 151; Email: fjtarazona@hospital-ribera.com

Abstract

Introduction: Osteoporotic hip fractures are important health problems in geriatric patients. The mortality rate can reach $10 \%$ during admission in hospital and 30\% after 12 months. A meta-analysis of 35 independent studies described greater survival among patients who underwent early surgery. Nevertheless, controversy remains about the acceptable waiting time for surgery. The objective of this study is to describe the new knowledge on the relationship between surgical delay and mortality.

Methods: The present review was carried out by conducting an electronic search in OVID (Medline and Embase) on hip fracture, mortality and surgical delay. The search was limited to publications in the last 5 years and in English and Spanish. A total of 73 articles were obtained, of which 14 were finally selected.

Results: Papers can be grouped into those that fund or not a relationship between surgical delay and mortality. Among the first, different authors described a strong association between mortality and complexity measured by a comorbidities index (Charlson Comorbidity Index or American Society of Anaesthesiologists' score). On the other hand, more studies have shown a strong association between time to surgery and mortality. A study among 42,230 patients used time as a continuous variable showing that wait time was associated with a greater risk of 30 day mortality and other complications.

Conclusion: Surgical delay is clearly linked to mortality risk in the different cut-points studied. Elderly patients admitted at hospital with hip fracture might be operated in the first 24 hours if the clinical conditions allow the surgery process.

\section{Introduction}

Osteoporotic hip fractures are important health problems in geriatric patients. A total of 1.3 million hip fractures was diagnosed in 1990 [1] and this figure is expected to increase to over 6 million by 2050 [2]. The mortality rate can reach $10 \%$ during admission in hospital and 30\% after 12 months [3,4]. In-hospital mortality rate is linked to surgical management [5]. A meta-analysis of 35 independent studies recruiting 191,873 patients described greater survival among patients who underwent early surgery (odds ratio [OR] 0.74; 95\% confidence interval [CI] 0.67-0.81; p<0.001) [6]. Previously, Vidan et al. [7] found delays in surgery to be associated with increased mortality from day 6 after a fracture. A meta-analysis of 16 observational studies $(n=13,478)$ showed surgery in the first 24 hours after hospital admission, versus in the first 72 hours, to reduce patient mortality [8]. The main cause of surgical delays is the lack of available operating rooms7. Patients whose surgery is delayed are exposed to inflammation and hypercoagulable states for longer than those whose surgery is not delayed $[8,9]$.

Clinical stabilization, based on clinical recommendations and guidelines, on the part of the orthogeriatric team can contribute to reduce such delays, increasing comorbidity diagnostic precision [10]. Nevertheless, controversy remains about the acceptable waiting time for treatment [11]. The proportion of patients receiving surgery within 36 hours is the quality-of-care indicator used in the United Kingdom, but hospital adherence ranges from $14.7 \%$ to $95.3 \% 12$. As well as, surgical delay have been frequently measured in days13 and compared to early and delayed groups14. 
Perhaps, by this reason, the results of different studies on the effect of surgical delay on mortality are contradictory. The objective of this mini-review is to summarize the new knowledge on the relationship between surgical delay and mortality in elderly patients with hip fracture.

\section{Methods}

The present review was carried out by conducting an electronic search in OVID (Medline and Embase) using the MeSH construction: [Hip fractures] AND ([Mortality] or "mortality" [Subheading]) and ([Operative Time] OR "surgical delay" OR "time to surgery") was used. The search was limited to publications in the last 5 years; in English and Spanish; and in human subjects. A total of 73 articles were obtained, of which 14 were finally selected. Some additional instructions were added for certain specific objectives where necessary. In 2 cases, supplementary information was obtained in the form of references of the selected articles. Details of the evaluation and selection process of the items are shown in Figure 1. The articles were selected by six investigators based on the following inclusion criteria: randomized clinical trials, cohort studies, casecontrol studies, observational studies, and before-after analyses; population: geriatric patients with proximal femoral fracture; and outcomes: short and long-term mortality. The exclusion criteria were letters to the Editor, case reports, articles with no available abstract or those with only the abstract published. All the articles were reevaluated by the authors of the review, and final inclusion was restricted to those of sufficient quality to afford information pertinent to the objectives of this review.

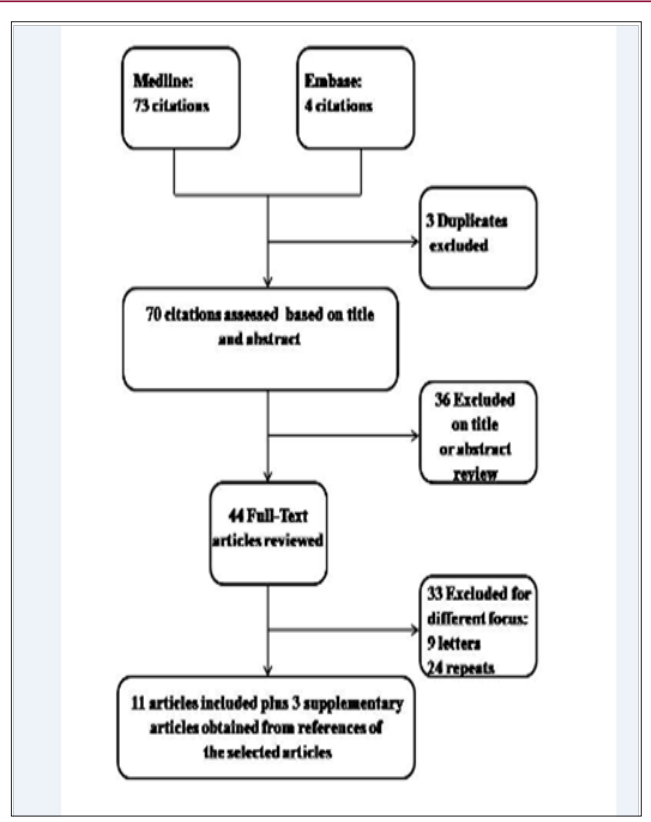

Figure 1: Flow chart study selection procedure used in literature search.

\section{Results}

Seventy three papers met the selection criteria. Of these, fourteen have been included in this mini-review. The authors have found works that can be grouped into those that do not find a relationship between surgical delay and mortality and those that do find this relationship. Among the first, different studies did not show any association between surgical delay and mortality. A prospective study on 628 patients with hip fracture did not show significant differences in in-hospital, 3-month or 1-year mortality between patients operated within 2 days and those operated after 3 or 4 days after hospital admission. However, there was significant higher 1-year mortality between patients operated within 4 days and those with surgery delayed more than 4 days [12]. A reason may be the different complexity of patients. The mean Charlson Comorbidity Index (CCI) score was significant higher among patients delayed more than 4 days. Excluding medically unfit for surgery patients at admission, there was also no significant difference at 3 -month or 1 -year mortality $(\mathrm{p}=0.384)$ between patients operated within 2 days and those with delay of surgery more than 2 days [12].

Different retrospective studies showed similar results. A retrospective study in 857 patients identified a relationship between the surgical delay and in-hospital mortality only when the time for surgery was higher than 96 hours [13]. In an observational study in 23,937 patients, the authors concluded than early surgery did not affect 30-day mortality after hospital discharge. Nevertheless, early surgery in patients with complex needs (CCCI score of 2 or higher) was associated with an increase in mortality. The authors considered than evidence appears unclear in the medical literature and the association between delay to surgery and perioperative mortality is still a questionable issue [14]. A study on 499 patients admitted with a diagnosis of intracapsular hip fracture did not show an association between in 1-year mortality and surgical delay. Time to surgery was divided in surgery before 24 hours of admission; surgery among 24 hours and 72 hours from admission; and surgery later than 72 hours [15]. The only variable than the authors could link to mortality was the American Society of Anaesthesiologists (ASA) classification of Physical Health score. Finally, a prospective cohort study of 6143 patients did not show any association between surgical delay and mortality [16].

On the other hand, more studies have shown a strong association between time to surgery and mortality. In a prospective observational study in 6638 patients, the authors used different thresholds ( 0 to 6 hours; 7 to 12 hours; 13 to 18 ; hours; 19 to 24 hours; 25 to 36 hours; 36 to 48 hours; and 48 to 72 hours) to know if time to surgery was linked to 30-day mortality. After adjusting for confounders (ASA score, age, type of fracture, function, cognitive situation and gender), surgery before 12 hours improved 1-month survival compared with surgery after 12 hours [17]. Other prospective cohort study on 418 patients showed than the ASA score and time to surgery were one-year mortality predictors in the final regression model. The authors concluded than surgical delay is a risk factor that has the potential to be modified to improve survival [18]. In a pre-/postintervention study (3525 pre- and 3007 postintervention patients), the authors observed than having surgery within 48 hours after hospital admission decreased risk of death in hospital (HR 0.51, 95\%CI 0.41-0.63) and at 1-year postsurgery (HR 0.72 , 95\% CI 0.64-0.80) after adjusting by age, 
sex, comorbidities, type of surgery and year [19]. A retrospective study in 3517 hip fracture surgeries shown an increased risk of thirty-day mortality with a surgical delay of more than twelve hours, than twenty-four hours and more than forty-eight hours and an increased risk of ninety-day mortality with a surgical delay of more than twenty-four hours. As well as the authors described an increased risk of 30-day and 90-day mortality with ASA score and male gender [20].

A population-based wait-time data study among 42,230 patients with hip fracture analyzed the relationship between time elapsed from hospital arrival to surgery (in hours) and mortality within 30 days. Compared with 13731 propensityscore matched patients who received surgery earlier, patients who received surgery after 24 hours had a significantly higher risk of 30-day mortality. Restricted cubic spline, logistic regression, and propensity score models were all adjusted for the same covariates: age, sex, year, income quintile, rurality, transfer from any health care institution, medical comorbidity, fracture and surgery type, Injury Severity Score, surgeon volume and experience, hospital volume and type, and surgery duration. The authors conclude than increased wait time was associated with a greater risk of 30-day mortality and other complications. A wait time of 24 hours may represent a threshold defining higher risk [21]. Another paper in 1448 consecutive patients showed that the surgery within the first 48 hours was associated with a decreased 1-year mortality rate.

In a logistic regression analysis, intervention between 48 and 72 hours was not reported as a risk factor for mortality, both compared to early surgery (within 48 hours) and to late surgery (after 72 hours). The authors concluded that the option of operating within day 3 is not a valid alternative [22]. Other prospective cohort study analyzed the relationship among 1-year survival and mortality predictors. 252 patients were included and surgical delay, measured in days, had a hazard ratio for mortality of 1.07 (95\% Confidence interval= 1.03-1.12) adjusting by gender, age and ASA score [23]. Finally, a retrospective database analysis on 2, 121, 215 patients undergoing surgical repair of hip fracture showed that compared to same-day surgery, each additional day of delay was associated with a significantly higher complication rate. However, next-day surgery was not associated with an increased risk of in-hospital mortality. Surgery 2 calendar days (odds ratio: 1.13) and 3+ days (odds ratio: 1.33) after admission was associated with higher mortality rates. Surgical delay in hip fracture care contributes to patient morbidity and mortality [24].

\section{Discussion}

Although some clinical guidelines recommend surgical intervention in the first twenty-four hours after hospital admission for hip fracture, the results found in the review are contradictory [25-27]. Five studies included [11-15] did not find any association between time to surgery and mortality or they found this association after an important surgical delay (more than 96hours) [12,13]. Excepted a study with 23,937 patients [14], the rest of the studies have small samples and they emphasize the complexity of the case (estimated by ASA or CCI) than the surgical delay. Nevertheless, seven studies [17-24] did describe a link between time to surgery and short and long-term mortality adjusting by confounder variables. As well as, this higher risk of mortality associated with surgical delay is linked to higher risk of complications too [17]. In fact, one of the studies analyzed by the first time the surgical delay as a continuous variable in hours [21] describing an association between time to surgery and complications and death.

The authors of this study argued than a wait time of 24 hours may represent a threshold defining higher risk because complications increased when surgery was delayed after this time, irrespective of the complication, follow-up period, or patient subgroup assessed [21]. As well as, surgical delay higher than 24 hours increase the risk of wound infection (OR=3.48 (95\%CI 1.110.8) [28] and a prospective study on 568 patients described an association between surgical delay and hospital length of stay and complications [29]. This result agrees with the conclusions of a previous meta-analysis [6] that shown a reduced risk of mortality in patients underwent surgery in the first 24 hours after hospital admission. As Vidán et al. [7] shown, the second cause to delay the surgery was an acute medical condition and, by this reason, the orthogeriatric approach have shown a reduction in complication rate and mortality during hospital admission [30]. The conclusion of this bibliographic review is that surgical delay is clearly linked to mortality risk in the different cut-points studied. Elderly patients admitted at hospital with hip fracture might be operated in the first 24 hours if the clinical conditions allow the surgery process.

\section{References}

1. Johnell O, Kanis JA (2004) An estimate of the worldwide prevalence, mortality and disability associated with hip fracture. Osteoporos Int 15(11): 897-902.

2. Cooper C, Campion G, Melton LJ (1992) Hip fractures in the elderly: a worldwide projection. Osteoporos Int 2(6): 285-289.

3. Roche JJ, Wenn RT, Sahota O (2005) Effect of comorbidities and postoperative complications on mortality after hip fracture in elderly people: prospective observational cohort study. BMJ 331(7529): 1374.

4. Morris AH, Zuckerman JD (2002) AAOS Council of Health Policy and Practice, USA. American Academy of Orthopaedic Surgeons. National Consensus Conference on Improving the Continuum of Care for Patients with Hip Fracture. J Bone Joint Surg Am 84A(4): 670-674.

5. Della Rocca GJ, Crist BD (2013) Hip fracture protocols: what have we changed? Orthop Clin North Am 44(2): 163-182.

6. Moja L, Piatti A, Pecoraro V (2012) Timing matters in hip fracture surgery: patients operated within 48 hours have better outcomes. A meta-analysis and meta-regression of over 190,000 patients. PLoS One 7(10): e46175.

7. Vidán MT, Sánchez E, Gracia Y, Jose A sera (2011) Causes and effects of surgical delay in patients with hip fracture: a cohort study. Ann Intern Med 155(4): 226-233.

8. Beloosesky Y, Grinblat J, Pirotsky A, Weiss A, Hendel D (2004) Different C-reactive protein kinetics in post-operative hip-fractured geriatric patients with and without complications. Gerontology. 2004 Jul-Aug 50(4): 216-222.

9. Beloosesky Y, Hendel D, Weiss A, Hershkovitz A, Grinblat J, et al. (2007) Cytokines and C-reactive protein production in hip-fracture-operated elderly patients. J Gerontol A Biol Sci Med Sci 62(4): 420-426.

10. Simunovic N, Devereaux PJ, Sprague S (2010) Effect of early surgery after hip fracture on mortality and complications: systematic review and meta-analysis. CMAJ 182(15): 1609-1616. 
11. Pioli G, Barone A, Mussi C (2014) The management of hip fracture in the older population. Joint position statement by Gruppo Italiano Ortogeriatria (GIOG). Aging Clin Exp Res 26(5): 547-553.

12. Lizaur Utrilla A, Martinez Mendez D, Collados Maestre I, Miralles Muñoz FA, Marco-Gomez L, et al. (2016) Early surgery within 2 days for hip fracture is not reliable as healthcare quality indicator. Injury 47(7): 1530-1535.

13. Major LJ, North JB (2016) Predictors of mortality in patients with femoral neck fracture. J Orthop Surg (Hong Kong) 24(2): 150-152.

14. Forni S, Pieralli F, Sergi A, Lorini C, Bonaccorsi G, et al. (2016) Mortality after hip fracture in the elderly: The role of a multidisciplinary approach and time to surgery in a retrospective observational study on 23,973 patients. Arch Gerontol Geriatr 66: 13-17.

15. Crego Vita D, Sanchez Perez C, Gomez Rico JA, de Arriba CC (2017) Intracapsular hip fractures in the elderly. Do we know what is important? Injury 48(3): 695-700.

16. Lund CA, Moller AM, Wetterslev J, Lundstrom LH (2014) Organizational factors and long-term mortality after hip fracture surgery. A cohort study of 6143 consecutive patients undergoing hip fracture surgery. PLoS One 9(6): e99308.

17. Bretherton CP, Parker MJ (2015) Early surgery for patients with a fracture of the hip decreases 30-day mortality. Bone Joint J 97B(1): 104108.

18. Ribeiro TA, Premaor MO, Larangeira JA, Brito LG, Luft M, et al. (2013) Predictors of hip fracture mortality at a general hospital in South Brazil: an unacceptable surgical delay. Clinics (Sao Paulo) 69(4): 253-258.

19. Bohm E, Loucks L, Wittmeier K, Lix LM, Oppenheimer L (2015) Reduced time to surgery improves mortality and length of stay following hip fracture: results from an intervention study in a Canadian health authority. Can J Surg 58(3): 257-263.

20. Nyholm AM, Gromov K, Palm H, Brix M, Kallemose T, et al. (2015) Danish Fracture Database Collaborators. Time to Surgery Is Associated with
Thirty-Day and Ninety-Day Mortality After Proximal Femoral Fracture: A Retrospective Observational Study on Prospectively Collected Data from the Danish Fracture Database Collaborators. J Bone Joint Surg Am 97(16): 1333-1339.

21. Pincus D, Ravi B, Wasserstein D, Huang A, Paterson JM, et al. (2017) Association Between Wait Time and 30-Day Mortality in Adults Undergoing Hip Fracture Surgery. JAMA 318(20):1994-2003.

22. Rosso F, Dettoni F, Bonasia DE, Olivero F, Mattei L, et al. (2016) Prognostic factors for mortality after hip fracture: Operation within 48 hours is mandatory. Injury 47(Suppl 4): S91-S97.

23. Campos S, Alves SM, Carvalho MS, Neves N, Trigo Cabral A, et al. (2015) Time to death in a prospective cohort of 252 patients treated for fracture of the proximal femur in a major hospital in Portugal. Cad Saude Publica 31(7): 1528-1538.

24. Ryan DJ, Yoshihara H, Yoneoka D, Egol KA, Zuckerman JD (2015) Delay in Hip Fracture Surgery: An Analysis of Patient-Specific and HospitalSpecific Risk Factors. J Orthop Trauma 29(8): 343-348.

25. (2018) ACS TQIP Best Practices in Management of Orthopaedic Trauma 2018.

26. Canadian Institute for Health Information (2017) Wait times for priority procedures in Canada.

27. Royal College of Physicians (2015) National Hip Fracture Database Annual Report 2015. London, England: Royal College of Physicians.

28. Cordero J, Maldonado A, Iborra S (2016) Surgical delay as a risk factor for wound infection after a hip fracture. Injury 47(Suppl 3): S56-S60.

29. Mariconda M, Costa GG, Cerbasi S, Recano P, Aitanti E, et al. (2015) The determinants of mortality and morbidity during the year following fracture of the hip: a prospective study. Bone Joint J 97B(3): 383-390.

30. Grigoryan KV, Javedan H, Rudolph JL (2014) Orthogeriatric care models and outcomes in hip fracture patients: a systematic review and metaanalysis. J Orthop Trauma 28(3): e49-e55.

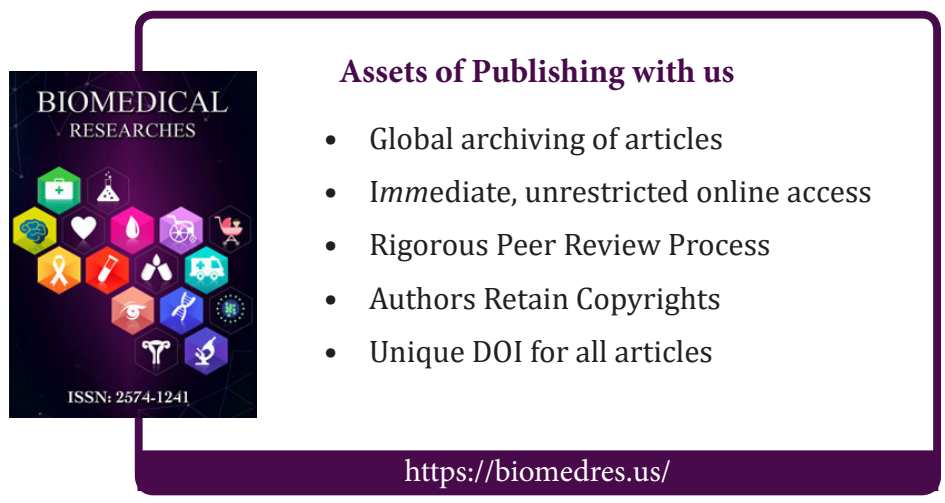

